\title{
Solar cycle prediction using precursors and flux transport models
}

\author{
R. Cameron and M. Schüssler \\ Max Planck Institut für Sonnensystemforschung, Max-Planck-Str. 2, 37191 \\ Katlenburg-Lindau, Germany \\ cameron@mps.mpg.de, schuessler@mps.mpg . de
}

\begin{abstract}
We study the origin of the predictive skill of some methods to forecast the strength of solar activity cycles. A simple flux transport model for the azimuthally averaged radial magnetic field at the solar surface is used, which contains a source term describing the emergence of new flux based on observational sunspot data. We consider the magnetic flux diffusing over the equator as a predictor, since this quantity is directly related to the global dipole field from which a Babcock-Leighton dynamo generates the toroidal field for the next activity cycle. If the source is represented schematically by a narrow activity belt drifting with constant speed over a fixed range of latitudes between activity minima, our predictor shows considerable predictive skill with correlation coefficients up to 0.95 for past cycles. However, the predictive skill is completely lost when the actually observed emergence latitudes are used. This result originates from the fact that the precursor amplitude is determined by the sunspot activity a few years before solar minimum. Since stronger cycles tend to rise faster to their maximum activity (known as the Waldmeier effect), the temporal overlapping of cycles leads to a shift of the minimum epochs that depends on the strength of the following cycle. This information is picked up by precursor methods and also by our flux transport model with a schematic source. Therefore, their predictive skill does not require a memory, i.e., a physical connection between the surface manifestations of subsequent activity cycles.
\end{abstract}

Subject headings: Sun: magnetic fields — Sun: photosphere

\section{Introduction}

A prediction of the strength of future solar cycles is of interest because a) expected future levels of solar activity have implications for space missions and for foreseeing potential 
hazards due to 'space weather', and b) it can be used to test theoretical models of the solar cycle, if the prediction is based upon a physical approach. Existing attempts to predict solar activity levels can be broadly divided into two groups: 1) extrapolation models, deriving a prediction from a purely mathematical analysis of the past records of solar activity, for instance by harmonic analysis (e.g., Rigozo et al. 2001; Echer et al. 2004) or by using concepts from nonlinear dynamics (e.g., Sello 2001), and 2) precursor models based upon correlations between certain measured quantities in the declining phase of a cycle and the strength of the next cycle (e.g., Hathaway et al. 1999; Schatten 2003). While the overall success of these models in predicting the future has been rather limited (e.g., see Figure 14.2 in Wilson 1994 and Figure 6 of Lantos \& Richard 1998), a number of methods have demonstrated considerable skill in 'predicting' the strength of the past solar cycles (e.g. Hathaway et al. 1999). In particular, some methods based on the level of geomagnetic variations (as measured, e.g., by the $a a$ or $A_{p}$ indices) a few years before and around sunspot minimum provide high correlation coefficients of up to 0.97 between the respective predictor and past maxima of the sunspot record (e.g., Legrand \& Simon 1981; Layden et al. 1991; Thompson 1993; Lantos \& Richard 1998; Hathaway et al. 1999)

Since the recurrent geomagnetic variations in the late phases of a cycle are presumably dominated by the fast solar wind streams from the equatorward expanding polar coronal holes of the Sun, some relation to the strength of the polar magnetic field during that phase has been suggested. As noted by, e.g., Schatten et al. (1978) and (Layden et al. 1991), such a relationship would be generally consistent with the Babcock-Leighton type of dynamo models for the solar cycle (Babcock 1961; Leighton 1969; Giovanelli 1985; Wang \& Sheelev 1991; Choudhuri et al. 1995; Durney 1995, 1996; Dikpati \& Charbonneau 1999; Küker et al. 2001). Such models assume that the differential rotation winds up a dipolar global poloidal field which is thought to dominate the large-scale surface field distribution around solar minimum and creates sub-surface toroidal fields, whose later eruption causes the magnetic activity of the following cycle. The larger the global dipole moment is, the stronger the toroidal field becomes and, presumably, the stronger the next cycle will be. In the course of the cycle, the global dipole field is reversed and an opposite-polarity field built up, owing to the preferential poleward drift of the follower polarity parts of solar active regions and the diffusion and cancellation of preceding-polarity flux over the equator. This occurs because there is a (statistically) systematic tilt angle of the bipolar magnetic regions with respect to the equator (Joy's law). The important point is that the mechanism which provides the poloidal field for the next cycle operates in the near-surface layers and thus is directly accessible to observation while the old cycle is still ongoing. If quantitatively understood this could provide a physical basis for prediction and, at the same time, a testbed for dynamo models. 
The first attempt to actually use a theoretical dynamo model for predicting future solar activity levels was presented recently by Dikpati et al. (2006) and Dikpati \& Gilman (2006), in the following referred to as the DDG model. They considered a Babcock-Leighton type flux-transport dynamo with solar-like differential rotation, advection of the magnetic field by a prescribed meridional circulation pattern (poleward near the surface, equatorward in the lower convection zone) and two sources for the poloidal field: one near the bottom of the convection zone and one resulting from active-region tilt near the surface. Parametrizing the near-surface source with the observed record of sunspot areas since 1874 and making simple assumptions concerning the tilt angle and the equatorward migration of the source latitude (corresponding to the butterfly diagram), they took the amount of flux of the low-latitude toroidal field generated by the model as a measure of predicted solar activity. Their model is able to reproduce the variation of the amplitudes of cycles 16-23 remarkably well, with correlation coefficients reaching up to nearly 0.99 , depending on the model parameters.

The success of the DGG model suggests that it may have captured the essential ingredients of the solar dynamo mechanism and that the dynamo is of the Babcock-Leighton flux-transport type. This would be an enormous advance in our understanding and, therefore, the evidence must be evaluated thoroughly and critically. We have therefore set out to answer the following questions: 1) Which are the key physical features that underly the predictive skill of the the DGG model? 2) How much of the predictive skill is already contained in the surface transport part? 3) Could the predictive skill be affected by correlations in the sunspot record (used as input data for the model) that are not specific to any particular dynamo model?

In order approach answers to the first two questions, we have carried out an exploratory study with a simple surface flux transport model. Two-dimensional versions of such models have successfully reproduced the observed evolution of the solar surface field (e.g., Wang et al. 1989a, b; van Ballegooijen et al. 1998; Schrijver 2001; Durrant et al. 2004; Baumann et al. 2004). For simplicity (and consistent with the surface part of the DGG model) we have considered azimuthally averaged quantities in a one-dimensional flux transport model and determined the evolution of the latitude-dependent surface field on the basis of observational input data (sunspot groups or sunspot numbers). If the generation of the poloidal field for the next cycle actually takes place near the surface in the fashion exhibited by the Babcock-Leighton approach, we should be able to find relevant predictors from such results. We have therefore compared quantities related to the reversal and built-up of the global dipole field, particularly the amount of flux diffusing and cancelling over the equator, with the activity of the following cycle.

In order to address the third question, we have also studied whether the predictive skill 
of the precursor-type models (including our own and possibly also DDG model) could be affected by the overlap of consecutive cycles and the resulting shift of the epoch of activity minimum depending on the strength and the rise time of the following cycle.

The paper is organized as follows. We describe the flux transport model in Sect. 2 and define the quantities considered as predictors in Sect. 3, Results based upon observational datasets for sunspot areas and sunspot numbers are shown in Sect. 4. We first closely follow the procedure used by DDG and compare with their results. Then we evaluate the sensitivity of the results to parameter variations and to modifications of the model assumptions concerning the input data, particularly the latitude distribution of emerging flux. In Sect. 5 we consider series of synthetic cycles with random amplitudes in order to study the effect of the amplitude-dependent shift of the activity minimum epoch on prediction methods. Sect. 6 contains a discussion of the results and gives our conclusions.

\section{A simple flux transport model}

We model the evolution of the (azimuthally averaged) component of the field normal to the solar surface, $B_{r}$, as a function of latitude, $\lambda$, and time, $t$. We assume that the surface flux is passively advected by a meridional flow, $v(\lambda)$, and by supergranulation, the latter being described as a turbulent diffusion process with an effective diffusivity $\eta$. In addition, we have a source term, $S(\lambda, t)$, describing the emergence of new bipolar magnetic regions at the surface, and an exponential decay term with a characteristic time scale $\tau$, which roughly

mimics 3-D effects (Schrijver et al. 2002; Baumann et al. 2006). Accordingly, our simple 1-D surface transport equation is written as

$$
\begin{aligned}
\frac{\partial B_{r}}{\partial t} & =\frac{1}{R_{\odot} \cos \lambda} \frac{\partial}{\partial \lambda}\left[v(\lambda) B_{r} \cos \lambda\right]+ \\
& +\frac{\eta}{R_{\odot}^{2} \cos \lambda} \frac{\partial}{\partial \lambda}\left(\sin \lambda \frac{\partial B_{r}}{\partial \lambda}\right)-\frac{B_{r}}{\tau}+S(\lambda, t) .
\end{aligned}
$$

For simplicity, we take $B_{r}$ to be antisymmetric with respect to the equator $(\lambda=0)$ and consider only the Northern hemisphere, thus ignoring North-South asymmetries.

We solve this 1D linear advection-diffusion problem by a fully implicit finite difference scheme with 900 grid cells per hemisphere. Since the bipolar regions described by the source term typically have a size of a few degrees, all features occurring in the simulation are very well resolved. In fact, tests with only 90 grid points give essentially the same results. The timestep of the numerical integration is set according to the Courant-Friedrichs-Levy condition on the basis of the maximum meridional flow speed. Values typically are of the 
order of a day.

\section{Predictors}

Which quantities provided by the surface flux transport model could be considered as potential predictors for the strength of the next cycle? In the framework of Babcock-Leighton dynamos, one is tempted to use the maximum value of the reversed polar field built up after solar maximum as an indicator for the strength of the poloidal field from which the toroidal field of the next cycle is being generated by differential rotation (Schatten et al. 1978). In fact, (Svalgaard et al. 2005) have reported a correlation between observed polar fields and sunspot activity of the subsequent cycle, but regular measurements of the polar fields exist only since about 1970 and still are rather uncertain and noisy (see, e.g., Arge et al. 2002). Furthermore, Layden et al. (1991) have tried to extend the temporal basis by considering potential proxies for the polar field such as the shape of the polar corona, coronal holes, or polar faculae, but found no clear evidence of a 'predictive' skill.

Even in the framework of the Babcock-Leighton scenario, it is not so clear whether the maximum polar field strength necessarily represents the relevant quantity that determines the strength of the next cycle. In a flux transport dynamo, the amount of poloidal magnetic flux reaching deep into the convection zone (the global dipole) is relevant for the generation of the toroidal field emerging during the next cycle. The actual polar surface field, on the other hand, contains also more superficial flux which is still topologically connected with the corresponding preceding-polarity flux on the same hemisphere. Furthermore, highlatitude flux emergence could possibly also contribute to the polar field (Durrant et al. 2002). The reversal and built-up of the global dipole field relevant for a flux-transport dynamo is determined by the amount of preceding-polarity flux that diffuses across the equatorial plane and reconnects with the opposite-polarity preceding flux on the other hemisphere. This is nicely illustrated by Figure 10 of Dikpati \& Gilman (2006). We therefore consider the amount of flux crossing the equator (per unit time, or time-integrated) as the potentially most relevant precursor for the strength of the subsequent cycle in the framework of a Babcock-Leighton dynamo. It can easily be calculated in surface flux transport simulations

and it also lends itself to observational determination (Durrant et al. 2004). Note that this predictor differs from what is used by DGG, namely, the low-latitude toroidal flux at the bottom of the convection zone, which is not present in our model. However, in a linear Babcock-Leighton type flux transport dynamo, the generated toroidal field is directly related to the poloidal flux diffusing over the equator. Thus there is a physical link between the two models, although we do not claim that our model provides a direct test of the DGG model. 


\section{Results of the flux transport model}

We have considered two sets of simulation runs with our flux transport model. In the first set, the results of which are presented in Sect. 4.1, we have followed as closely as possible the procedures in the DDG model in order to study whether part of the predictive skill of their model is already contained in the surface flux transport. The second set of simulations addresses the dependence of the predictive skill on the parameters of the flux transport model as well as on the assumptions and procedures concerning the emergence latitudes of tilted bipolar regions.

\subsection{Input model according to Dikpati et al.(2006)}

The DDG model (Dikpati et al. 2006) is described in some more detail in Dikpati \& Gilman (2006). Excluding parameters of the dynamo model which are of no concern here, the procedures can be described as follows:

1. The surface source term for the poloidal magnetic field (more precisely, for the azimuthal component of the vector potential) is taken proportional to the sunspot areas given in the combined $\mathrm{RGO} / \mathrm{SOON}$ observational record provided by D. Hathaway (NASA/MSFC)1.

2. The individual solar cycles are stretched or compressed in time to fit with a mean cycle length of $10.75 \mathrm{yr}$.

3. The source term has a Gaussian latitudinal profile with a fixed full width at half maximum (FWHM) of $6^{\circ}$, migrating equatorward between $35^{\circ}$ and $5^{\circ}$ between cycle minima.

4. The magnetic diffusivity in the near-surface layers has a value of about $300 \mathrm{~km}^{2} \mathrm{~s}^{-1}$.

5. The poleward meridional flow at the surface has the form $v(\lambda)=v_{0} \sin (2 \lambda)$ with $v_{0}=14.5 \mathrm{~m} \mathrm{~s}^{-1}$.

We have adapted our surface transport model as far as possible to these parameters and procedures. We describe the source term (in our case, written for $B_{r}$ ) by two Gaussians of opposite sign with a FWHM of $6^{\circ}$, centered at $\lambda_{0}(t) \pm 0 .{ }^{\circ} 5$, where $\lambda_{0}(t)$ parametrizes the

\footnotetext{
${ }^{1}$ http://solarscience.msfc.nasa.gov/greenwch.shtml
} 
equatorward drift of the flux emergence latitude between $35^{\circ}$ and $5^{\circ}$ as a linear function of cycle phase. The amplitude of the Gaussians as a function of time is taken to be proportional to the monthly averages of the sunspot area from the RGO/SOON dataset, smoothed by a 6 -month boxcar running mean. For the decay time, $\tau$, we use a value of 5.7 years (roughly corresponding to a volume diffusivity of about $100 \mathrm{~km}^{2} \mathrm{~s}^{-1}$, see Baumann et al. 2006).

The epochs of the sunspot minima have been taken from the National Geophysical Data Center (NOOA/NGDC)2. Since cycle 23 is still ongoing, we have considered three possible values for the epoch of the upcoming sunspot minimum, namely 2007.0, 2007.5 and 2008.0, respectively. Since the RGO/SOON data are available only until 10/2005, we have substituted the missing data between $11 / 2005$ and the assumed minimum epoch by data for the corresponding phase of cycle 22 .

Figure 1 shows the time evolution (for the period covered by the RGO/SOON data) of various quantities and the corresponding correlation diagrams. The top panels give the RGO/SOON sunspot areas and the correlations between the maximum of a cycle with that of the preceding cycle; obviously, the amplitude of the foregoing cycle is a poor predictor (correlation coefficient $r=0.47$ ). The second panel from the top shows the polar field as resulting from the flux transport simulation. It peaks typically around sunspot minimum and largely reflects the level of activity of the onqoing cycle, a common result of surface flux transport models (Schrijver et al. 2002; Wang et al. 2002, 2005; Baumann et al. 2006). Consequently, the predictive power is poor $(r=0.35)$.

The third panel shows the amount of magnetic flux diffusing over the equator per unit time, which we henceforth denote by $\Phi$, for simplicity. We consider this predictor to be related to the build-up of the global poloidal field relevant for the dynamo. We see that this quantity shows a reasonable predictive skill with $r=0.90$. In particular, the strong drop of activity from cycle 19 and 20 is correctly anticipated. On the other hand, the prediction for the relatively high cycle 21 is quite bad. Finally, the bottom panel gives the dipole component of the simulated surface field, which mainly results from the accumulated flux diffusing over the equator. It shows a predictive power that is comparable to that of $\Phi$ $(r=0.83)$.

As an alternative to correlating the maxima, we have also considered time-integrated quantities, namely, cycle-integrated sunspot area and magnetic flux diffused over the equator as well as polar field and dipole component of the surface field integrated between polarity reversals. All integrals have been determined for unstretched cycles, i.e., keeping the actual cycle lengths. The correlation coefficients of the integrated sunspot area of the subsequent

\footnotetext{
${ }^{2} \mathrm{ftp}$ //ftp.ngdc.noaa.gov/STP/SOLAR_DATA/SUNSPOT_NUMBERS/maxmin.new
} 
cycle with the integrated flux over the equator $(r=0.92)$ and the dipole component $(r=$ 0.82) are similar to the corresponding values for the maxima. Interestingly, the correlations coefficients for the integrated polar field and the sunspot area of the preceding cycle are much larger: 0.79 and 0.78 , respectively. At least part of this increase comes from the better 'prediction' of cycle 20, whose lower amplitude is largely compensated by a its longer duration in the integrals. This result indicates that all such correlations should be considered with great caution because they can be sensitive to the precise definition of the quantities considered and their real significance is difficult to evaluate.

The results presented in Figure 1 show that a significant part of the predictive skill contained in the DDG model is covered by surface quantities resulting from a simple flux transport model, although we do not quite reach such high correlation coefficients when using their model parameters. The fact that the magnetic flux diffusing over the equator appears to be a fairly good predictor would be consistent with its putatively important role played in a Babcock-Leighton dynamo. What is relevant for the amplitude $\Phi$ ? Most importantly, it is the amount of flux emerging near to the equator in the late phases of a cycle. Since the distance to the equator is small and since the poleward meridional flow accelerates away from the equator, low-latitude bipolar magnetic regions contribute most to $\Phi$. Figure 2 illustrates the importance of low-latitude emergence during the declining phase of the cycle for the predictor $\Phi$. Given are the observed sunspot area (solid) and the flux diffusing over the equator, $\Phi$ (dashed), for cycles 18, 19, and 20. In addition, the dotted lines (with a separate axis on the right hand side) show the linear progression of the centroid of the emergence latitude assumed in the model. The maxima of $\Phi$ are shifted by about 3-4 years with respect to the preceding sunspot maxima and occur about 2-3 years before the subsequent minima. Since the $\Phi$ curve essentially represents a convolution of the sunspot area curve with the latitude sensitivity of the flux diffusing over the equator (which is heavily weighted towards the low latitudes), its amplitude is determined by the sunspot area and the emergence latitude at a given time. The sunspot area is much larger during the time interval when the emergence latitude ranges between about $15^{\circ}$ and $5^{\circ}$ in the case of cycle 18 than for cycle 19. Consequently, the prediction for cycle 19 is much higher than that for cycle 20. This result already indicates that the predictor could be rather sensitive to the definition of the source latitudes in the model.

A note concerning the prediction for cycle 24: the results shown in Figure 1 are based on assuming 2007.0 for the epoch of the upcoming sunspot minimum. This yields a predicted maximum sunspot number of about 160 for cycle 24. However, a later minimum would lead to significantly lower predictions: about 130 for a 2007.5 minimum, and about 110 for a minimum at 2008.0. The first two values are consistent with the DDG prediction of a cycle 24 amplitude exceeding that of cycle 23 by $30-50 \%$, while the third case would predict an 
activity similar to that of cycle 23. All these results are affected in an unknown way by the substitution of data from cycle 22 for unknown cycle 23 data. In any case, we see that the predictions rather sensitively depend on the assumed epoch of the upcoming activity minimum - the significance of this result will become clear when we consider the origin of the predictive skill in Sect. 5 .

\subsection{Modifications of the procedure}

We have seen that the amount of preceding-polarity flux diffusing over the equator appears to represent a reasonably good predictor for the next cycle. In this section we will evaluate a) how strongly this result depends on parameter choices in our flux transport model and b) whether the predictive skill can be improved by using more of the observational information contained in the available sunspot data. It turns out that the correlation coefficients for the maxima of the polar field are always small, while the results for the dipole component of the surface field and for the flux crossing the equator, $\Phi$, are similar to each other in most cases. Therefore, in what follows we restrict ourselves to showing results for the latter quantity. 
Table 1. Correlation coefficients between the predictor, $\Phi$, and the strength of the following activity cycle

\begin{tabular}{|c|c|c|c|c|}
\hline variable & reference & $a$ & $b$ & $c$ \\
\hline 1. meridional flow velocity, $v_{0}\left(\mathrm{~m} \mathrm{~s}^{-1}\right)$ & $\begin{array}{l}14.5 \\
0.90\end{array}$ & $\begin{array}{l}10 . \\
0.88\end{array}$ & $\begin{array}{l}20 . \\
0.85\end{array}$ & \\
\hline 2. meridional flow profile & $\begin{array}{c}\mathrm{DDG} \\
0.90\end{array}$ & $\begin{array}{c}\text { SB2006 } \\
\text { 0.82 }\end{array}$ & & \\
\hline 3. magnetic diffusivity, $\eta\left(\mathrm{km}^{2} \mathrm{~s}^{-1}\right)$ & $\begin{array}{l}300 . \\
0.90\end{array}$ & $\begin{array}{l}100 . \\
0.70\end{array}$ & $\begin{array}{l}200 . \\
0.79\end{array}$ & $\begin{array}{l}600 . \\
0.76\end{array}$ \\
\hline 4. decay time, $\tau(\mathrm{yr})$ & $\begin{array}{l}5.6 \\
0.90\end{array}$ & $\begin{array}{l}1.7 \\
0.89\end{array}$ & $\begin{array}{l}27.8 \\
0.90\end{array}$ & \\
\hline 5. tilt factor in source amplitude & $\begin{array}{r}\text { none } \\
0.90\end{array}$ & $\begin{array}{r}\sin \lambda \\
0.88\end{array}$ & & \\
\hline 6. Gaussian width, FWHM (deg) & $\begin{array}{c}6 . \\
0.90\end{array}$ & $\begin{array}{c}3 . \\
0.85\end{array}$ & $\begin{array}{l}12 . \\
0.66\end{array}$ & \\
\hline 7. Latitude shift of Gaussians (deg) & $\begin{array}{c}1 . \\
0.90\end{array}$ & $\begin{array}{c}2 . \\
0.90\end{array}$ & $\begin{array}{c}3 . \\
0.90\end{array}$ & $\begin{array}{c}4 . \\
0.91\end{array}$ \\
\hline 8. Latitude range of emerging flux (deg) & $\begin{array}{r}5-35 \\
0.90\end{array}$ & $\begin{array}{r}1-31 \\
0.80\end{array}$ & $\begin{array}{l}5-25 \\
0.74\end{array}$ & $\begin{array}{r}5-40 \\
0.82\end{array}$ \\
\hline 9. stretching of cycles & $\begin{array}{l}\text { yes } \\
0.90\end{array}$ & $\begin{array}{l}\text { no } \\
0.91\end{array}$ & & \\
\hline
\end{tabular}

Note. - The first column indicates the variable or procedure of the model being varied. The subsequent columns show the results for the reference case (Sect. 4.1) and the variations of the model. In each case, the upper number gives the value of the parameter under consideration and the lower number (in italics) the gives corresponding correlation coefficient. 
As a first step, we consider the effect of parameter variations in our model on the correlation coefficient between the maximum of $\Phi$, the amount of flux diffusing over the equator, and the activity maximum of the subsequent cycle. The basic procedure for including the emerging flux as a source in the model is the same as in the reference case discussed in the preceding section. We have varied the following parameters:

1. Peak meridional velocity, $v_{0}$.

2. Latitude profile of the meridional flow, $v(\lambda)$. As an alternative to the profile used by DDG, we consider a semi-empirical profile adapted to helioseismic measurements, $v(\lambda)=1.6 \sin (2 \lambda) \exp \left[\pi\left(1-|\lambda| / 90^{\circ}\right)\right]$, in units of $\mathrm{m} \mathrm{s}^{-1}($ Schüssler \& Baumann 2006).

3. Turbulent magnetic diffusivity, $\eta$. The value of $600 \mathrm{~km}^{2} \mathrm{~s}^{-1}$ favored by surface fluxtransport models (e.g., Durrant \& Wilson 2003; Baumann et al. 2004) is a factor of 2 larger than the surface value considered by DDG. We have also considered smaller values of $\eta$.

4. Decay time, $\tau$. The results of Schrijver et al. (2002) and Baumann et al. (2006) favor a value between 5 and 10 years.

5. Tilt angle factor in the source amplitude. DDG have taken the amplitude of their source term proportional to the sunspot area as a proxy of the amount of emerging flux, thus implicitly assuming that the average tilt angle of the emerging bipolar regions is independent of latitude. In reality, the average tilt angle is roughly proportional to $\sin \lambda$ (e.g., Howard 1991). This can be included into the model by multiplying the source term by $\sin \lambda$. The linearity of the flux transport model permits us to ignore the factor of proportionality since it represents only a scaling factor that does not affect the correlation results.

6. FWHM of the Gaussians in the source term. DDG have assumed a fixed latitudinal width of $6^{\circ}$ for their source term, corresponding to a constant width of the activity belt in the course of the cycle.

7. Latitude shift between the Gaussians. As long as this shift is small compared to the width of the Gaussian, the sum of the two Gaussians (of opposite sign) used in our source term is proportional to the derivative of the Gaussian with respect to latitude and the width of the resulting source term is only determined by the FWHM of the individual Gaussians. The effect of moving away from this limit can be evaluated by increasing the shift from $1^{\circ}$ (reference case) to larger values. 
8. Range of emergence latitudes. The procedure of DDG assumes that the source progresses through a fixed latitude interval in the course of each cycle. The boundaries of this interval are adjustable parameters.

9. Stretching of cycles. In order to keep their dynamo model in phase with the source term derived from the actual sunspot data, DDG had to stretch (or compress) the lengths of the individual cycles to a fixed value of 10.75 years. Our simple model does not require such stretching, so that the actual cycle length can be used.

Table 1 gives the correlation coefficients between the maximum of $\Phi$ and the maximum sunspot area of the subsequent cycle for various parameter changes, following the sequence of the preceding list. The correlation coefficients are printed in italic numbers in the second row for each case. As reference we take the case presented in Sect. 4.1, which follows closely the DDG procedures. We see that the most sensitive parameters are the magnetic diffusivity and the latitudinal width of the source term, which is not surprising in view of the importance of these parameters with respect to our predictor, $\Phi$. The corresponding correlation coefficients based on the cycle-integrated quantities are very similar to those shown in Table 1 and thus need not to be discussed any further. Altogether, it is clear from the table that, as long as we keep the basic procedures used by DDG, the correlation coefficients are not strongly dependent on the parameters, with values around $r=0.90$ being common. We can easily obtain even higher values by performing some fine tuning: for instance, for $\eta=200 \mathrm{~km}^{2} \mathrm{~s}^{-1}$ and a latitude range of $5^{\circ}-25^{\circ}$ for the source term, we find a correlation coefficient of 0.95 between the maxima of $\Phi$ and the sunspot area. However, such a procedure is dubious for obvious reasons and we have not tried to further 'optimize' the values.

\section{Dependence on input data set}

The cross-calibration between the RGO and the SOON data to obtain a consistent data set spanning the whole time period from 1874 on is not trivial. Balmaceda et al. (2005) have recently considered an additional sunspot data set, the "Russian books", compiled from data obtained at observatories in the former USSR. Applying a careful cross-calibration, they have bridged the RGO and the SOON data by parts of this data set and thus obtained a consistent record of sunspot areas. In order to evaluate the dependence of the predictive skill on the input data set, we have taken these data as input for our flux transport model. In addition, we also have used the monthly sunspot numbers as provided by the Solar Influences Data 
Analysis Center (SIDC) 3 at the Royal Observatory of Belgium. Figure 3 shows the quantity $\Phi$ obtained with the surface flux transport model using the three data sets for cycles 12-23. All other parameters are the same as those in Sect. 4.1. As apparent from Figure 3, there are no big differences between the results for the various datasets. In all cases, the drop from cycle 19 to cycle 20 is predicted well while the prediction for cycle 21 is much too low. The correlation coefficients with the activity maxima of the next cycle are $r=0.87$ for the dataset of Balmaceda et al. (2005) and $r=0.73$ for the sunspot numbers, to be compared with $r=0.90$ for the reference case. The corresponding values for cycle-integrated quantities are $0.83,0.80$, and 0.92 .

The satisfactory predictive performance of the model with input from monthly sunspot numbers suggests that we can use this dataset to include more cycles. The result for cycles 1-23 is shown in Figure 4. The correlation between the maxima of the flux diffusing over the equator and the maxima of the sunspot number of the subsequent cycle is $r=0.80$. The two curves are maximally correlated for a forward time shift of the $\Phi$ curve by 6.8 yr.

\section{The relevance of the declining phase}

In order to illustrate the importance of the activity level during the declining phase, we consider as a predictor the average sunspot activity three years before the minima of the (unstretched) historical cycles. The result based on the sunspot areas for cycles 12-23 are shown in Figure 5, while sunspot numbers for cycles 1-23 are considered in Figure 6. In both figures, the predictor is shown by the diamond-shaped symbols. In order to illustrate the predictive skill, we have marked the corresponding activity level (multiplied by a factor 3) by circles at the times of the following activity maxima and connected them by dashed lines. The general trends and the significant drops of activity after cycles 4, 11, and 19 are reproduced in both cases. The corresponding correlation coefficients are $r=0.84$ for Figure 5 and $r=0.89$ for Figure 6. The values for using the activity level 2 years and 4 years before minimum are $r=0.89$ and $r=0.83$, respectively, for the sunspot numbers of cycles 1-23. Consequently, the predictive skill of this very simple precursor is comparable to the corresponding cases using the predictor $\Phi$ from the flux transport model. Together with the considerations presented in connection with Figure 2 this indicates that the level of activity in the declining phase of a cycle is underlying the predictive skill of our flux transport model when the source term is specified according to the DGG model.

\footnotetext{
${ }^{3} \mathrm{http}: / /$ sidc.oma.ce
} 


\section{Changes of the source model}

Since the existing sunspot data since 1874 do actually contain the areas and coordinates of each individual observed sunspot group, one can use this information to make the source term more realistic. We have done so in two steps, both based on the cross-calibrated sunspot group data set provided by Balmaceda et al. (2005).

1) In the first step, we change from the schematically prescribed fixed range and drift rate of the emergence latitudes to the average observed actual latitudes of the sunspot groups. This is done in the following way (cf. Figure 7). We average the latitudes of the individual sunspot groups that appeared within one month, weighted by group area. Since a sunspot group typically appears more than once in the data, we consider a group only at the day of its maximum area in order to avoid multiple counting. When cycles overlap around solar minimum, sunspot groups near the equator are attributed to the old cycle while those appearing in higher latitudes are considered to be part of the new cycle (red and blue dotted areas in Figure 7). For each cycle, the resulting monthly averages of the emergence latitudes (green curves in Figure 7), are fitted to a parabola (black lines). This procedure leads to a observationally based time-latitude profiles of flux emergence for each cycle, also allowing for the overlapping of cycles. Inspection of these parabolic profiles in Figure 7 shows that, for most cycles, they deviate strongly from the linear profiles between $35 \mathrm{deg}$ and $5 \mathrm{deg}$ assumed in the reference case. In fact, they are better represented by a linear profile between $25 \mathrm{deg}$ and $5 \mathrm{deg}$.

We have run the flux transport model with these emergence profiles, considering separately the sunspot areas for each cycle in the source amplitudes, so that overlapping cycles are properly accounted for. Also, the tilt-angle factor $\sin \lambda$ has been included in the source amplitudes. All other parameters are as in the reference case discussed in Sect. 4.1, Figure 8 shows the resulting time evolution of the predictor $\Phi$ together with the observed (total) sunspot areas. Although the general trend of the cycle amplitudes is roughly reproduced, the value of $r=0.75$ for the correlation coefficient between the maxima shows that the predictive skill is significantly diminished with respect to the reference case. If we avoid the parabolic fit and directly use the monthly weighted averages (green curve in Figure 7), the correlation coefficient drops to $r=0.43$. Likewise, omitting the monthly averages of the emergence latitudes and using a area-weighted direct parabolic fit through the emergence latitudes of the individual sunspot groups (red and blue dots in Figure 7), yields $r=0.45$. In both cases, the predictive skill is almost completely lost.

This leaves us with the surprising result that the predictive skill is strongly dimished or even almost lost when more detailed observational data are used for prescribing the source in the flux transport model. Moreover, the skill depends critically on the way that the 
emergence latitudes of the individual sunspot groups are averaged. These results become less puzzling when we consider in Figure 7 the broad distribution of the actual emergence latitudes for any given time: since the contribution to the predictor $\Phi$ depends sensitively on the emergence latitude, in the sense that near-equator emergence is much more strongly weighted, variations in the averaging procedure that lead to small changes in the low-latitude part of the averaged emergence latitudes used in the flux-transport model can have a strong impact on the predictor quantity, $\Phi$.

2) We can avoid the sensitivity of the results with respect to the averaging procedure of the emergence latitude by directly considering the contribution to the source term of each individual sunspot group, so that no averaging is required. Each sunspot group in the data is identified with a bipolar magnetic region that is introduced into the flux transport simulation at its recorded latitude. The magnetic flux content of the region is assumed to be proportional to its maximum sunspot area. The orientation of the magnetic polarities follows Hale's laws and thus alternates from cycle to cycle. Taking the latitude separation of the two polarities of the region to be small compared to their diameter, which itself is assumed to be proportional to the square root of the sunspot area, we choose for the FWHM of the two Gaussians describing the radial field source

$$
\mathrm{FWHM}=\left(\frac{A_{\mathrm{s}}}{A_{\mathrm{s}, \max }}\right)^{1 / 2} \times 5^{\circ},
$$

where $A_{\mathrm{s}}$ is the area of the sunspot group and $A_{\mathrm{s}, \max }$ the value for the largest sunspot groups in the dataset. For these groups, we assume a latitude extension of $5^{\circ}$, roughly corresponding to half the size of the largest groups. It turns out that the the results are rather insensitive to the exact value of this parameter (see below). To account for the latitude dependence of the tilt angle (Howard 1991), the amplitude of the corresponding source term is multiplied by a factor $\sin \lambda$.

Figure 9 shows the prediction results. It is obvious that the predictor, $\Phi$, now mainly reflects the strength of the ongoing cycle and thus provides very low predictive capability. Accordingly, the correlation coefficient between the maxima of $\Phi$ and the sunspot area of the subsequent cycle is low: $r=0.33$ (the corresponding values for maximum latitude extensions of $10 \mathrm{deg}$ and $2.5 \mathrm{deg}$ are $r=0.27$ and $r=0.34$, respectively. The origin of this result is in the high sensitivity of $\Phi$ to bipolar regions emerging in low latitudes (cf. Figure2). Although the tilt angle goes to zero, $\Phi$ is still dominated by these low-latitude emergences. Since we have a broad distribution of emergence latitudes at any given time, there is nearly always some flux emergence in low latitudes (cf. Figure 7. Because the amount of this low-latitude flux emergence is mainly determined by the overall strength of the ongoing cycle, the quantity $\Phi$ is no longer dominated by the late phase as in the reference case, which did not take 
into account the broad latitude range of emerging flux. Consequently, the predictor mainly reflects the ongoing cycle, so that the predictive skill of the model is a nearly completely lost. Note that this version of the model makes the most direct use of the actual data.

A result pointing in this direction appears already in our parameter study (see Table 1): when doubling the latitudinal width of the source term (to 12 degrees FWHM of the corresponding Gaussian), the correlation coefficient dropped from 0.90 to 0.66 . When we impose a narrow latitudinal extension of the source term, low-latitude emergence occurs exclusively in the declining phase of a solar cycle, i.e., during a few years before sunspot minimum. The more activity (flux emergence) during the this phase, the more flux diffuses over the equator, and the higher is the amplitude of our predictor (cf. Figure 2).

\section{What is the origin of the predictive skill?}

We have seen that our predictor $\Phi$ determined with the flux transport model provides reasonable predictive skill in the case of a narrow latitudinal width and a prescribed fixed latitude range of the source term and we have shown that this is related to the activity level in the declining phase of the cycle. However, the predictive skill of $\Phi$ is strongly dimished or even nearly completely lost when averages of the actually observed observed emergence latitudes are considered or if the individual emergence latitudes of sunspot groups are used directly. This result casts serious doubt upon connecting the predictive skill in the standard case with the Babcock-Leighton dynamo scheme and with the dipole strength of the surface field. But how else can we understand the unquestionable correlation between the activity level in the declining phase of a cycle and the strength of the next cycle?

We suggest that in fact no direct physical link between the surface manifestations of the old and the new cycle is required to understand the predictive skill of the activity level in the declining phase. All that is needed are two well-established properties of the sunspot record: 1) the temporal overlapping of cycles with high-latitude spots of the new cycle already appearing when the old cycle is still in progression in low latitudes (e.g., Harvey 1992) and 2) the relation between the rise time of a cycle towards its maximum and its strength: stronger

cycles rise faster towards sunspot maximum (known as the Waldmeier effect, e.g. Waldmeier 1935). When considering latitude-integrated quantities like the sunspot number or area, the combined effect of both properties leads to a systematic shift of the minimum epochs between two cycles of different strength: the minimum occurs earlier if the following cycle is stronger than the preceding cycle and later for a weaker following cycle. This effect explains the empirical statistical relationship between cycle length and amplitude: strong cycles tend to be preceded by short cycles (e.g., Hathaway et al. 1999, 2002; Solanki et al. 2002). 
Figure 10 illustrates how the overlapping of cycles with amplitude-dependent rise time leads to a predictive skill of the activity level during the declining phase of the preceding cycle. In this figure we have considered time profiles of the activity cycles according to a prescribed functional form (Li 1999; Hathaway et al. 1994), which reproduces both the rise and decay parts of a cycle, including the Waldmeier effect:

$$
f(t)=\frac{a\left(t-t_{0}\right)^{3}}{e^{\left(t-t_{0}\right) / b}-c},
$$

where $t_{0}$ denotes the starting time of the cycle. The parameter $b=1.128 \mathrm{yr}$ and the relation between $c$ and $a$ of the form

$$
c=-1.10410^{-4} a^{2}+0.24666 a-123.593
$$

have been determined by Li (1999) on the basis of sunspot area data since 1876. By fitting the two parameters, $t_{0}$ and $a$, the cycles 12-22 are well reproduced by the functional form given by equation (3). The left panel of Figure 10 shows the summed activity levels of a cycle followed by a stronger cycle (solid curve) or by a weaker cycle (dashed curve), respectively, the follower cycle starting 11 years after the first cycle in both cases. The individual cycles profiles are indicated by the dotted lines. The faster rise of the stronger following cycle leads to a earlier 'sunspot minimum' than in the case of a weaker follower (marked in the figure by 'M1' and 'M2', respectively), the time shift being about one year. Since observationally a sunspot cycle is defined as the time between adjacent minima, the activity in the declining phase of the first cycle, (i.e., in a fixed time interval relative to the respective solar minimum epoch) is considerably larger when the follower cycle is stronger than when it it weaker (Hathaway et al. 1999). In our example, for instance, the activity level three years before the respective minimum is about a factor of two larger for the stronger following cycle than for the weaker follower (marked in the figure by 'P1' and 'P2'), corresponding to the relative strength of the two follower cycles. Consequently, the activity level at time P1/P2 can be used as a predictor for the amplitude of the following cycles, but it can only be determined after the epoch of the minimum is known; before, only upper limits can be given.

The left panel of Fig. 10 shows that the above defined predictor P1/P2 (activity level three years before cycle minimum) is only weakly affected by the strength of the first cycle. This results from the fact that the declining phase of a cycle largely represents an exponential decay, irrespective of the cycle strength. Fig. 10also suggests that the shift of sunspot minima depending on the amplitudes of overlapping cycles leads to a correlation of the activity level at sunspot minimum with the amplitude of the following cycle. In fact, Hathaway et al. (2002) find a correlation coefficient of $r=0.72$ between these quantities for the historical sunspot record. 
As a consequence, simply two well-known properties of the solar cycles, the Waldmeier effect and the overlapping, explain the predictive skill of the activity level in the declining phase of a cycle. This explanation also carries over to the skill of the our predictor from the flux transport model, the amount of flux diffusing over the equator: assuming for the source term a linear profile of emergence latitude versus time between two sunspot minima and an amplitude proportional to the instantaneous activity level, leads to a stronger source in low latitudes (declining phase of the cycle) for an earlier minimum, which, in turn, results from a stronger following cycle. Through the dependence of the sunspot minimum shift on the amplitude of the next cycle, information about the strength of the following cycle propagates into our predictor, completely independent of whether there is a physical connection between surface manifestations of the cycles or not. No further 'memory' of the system is required.

We demonstrate the effect of the minimum shift on the prediction with a precursor method by considering series of overlapping synthetic cycles with profiles according to equation (3). The cycle amplitudes form a random sequence with a flat probability density between 500 and 3000 microhemispheres for the maximum sunspot area, so that the strengths of subsequent cycles are completely independent of each other. The cycles start at regular intervals of $10.75 \mathrm{yr}$; because of their overlapping, the cycle length defined by the time between subsequent activity minima varies. As an example, Figure 11 shows the analog to Figure 6 for one realization of 23 random synthetic cycles. Statistics of this synthetic series, such as the correlation of the minima with the amplitudes of the subsequent cycle $(r=0.60)$ and the correlation between the length of a cycle and the amplitude of the next cycle $(r=-0.82)$ are roughly consistent with the properties of the actual sunspot record (exhibiting values of 0.72 and -0.69 , respectively, see Hathaway et al. 1999), which is sufficient for the purpose of illustration. Using the activity level 3 years before the activity minima as predictor, we find about the same skill for the synthetic random series (correlation coefficient $r=0.84$ ) as for the actual data $(r=0.89$, see Figure 6$)$. This demonstrates that the amplitude-dependent overlapping of cycles affects the timing of the activity minima in such a way that information from cycle $n+1$ can be picked up by considering the activity in the declining phase of cycle $n$, but only after the epoch of the minimum is known. As we have seen, this effect allows us to 'predict' a random sequence of cycle amplitudes without any relation between subsequent cycles apart from overlapping.

For 1000 random series of 8 cycles each, Figure 12 shows the cumulative probability distribution for the correlation coefficient between the activity level three years before minimum and the subsequent maximum. The median value corresponds to $r=0.83$, i.e., in $50 \%$ of the cases the correlation coefficient is larger than this value; it is larger than 0.95 in $5 \%$ of the cases. These results with random sequences of cycle amplitudes indicate that correlation coefficients in the range of $0.8-0.9$, which are regularly found with precursor methods, may 
be easily be explained by the minimum shift effect alone and require no further physical connection of the surface quantities between subsequent cycles. This does not mean that such a connection may not exist, only that correlations of that size do not compel us to assume such a connection.

\section{Discussion and conclusions}

The DDG model is able to reproduce the amplitudes of cycles 16-23 with impressively large correlation coefficients (up to $r \simeq 0.99$ ) and we have seen that our very much simpler model exhibits somewhat less (up to $r \simeq 0.95$ ), but still considerable predictive skill, provided that we closely follow the DDG treatment of the surface source term. In fact, for a BabcockLeighton type flux-transport dynamo, our predictor, the flux diffusing over the equator determines the strength of the reversed global dipole field from which differential rotation generates the toroidal flux for the next activity cycle. Therefore, it is tempting to consider these correlations as indicating that such a type of dynamo model in fact represents the engine underlying the solar activity cycle.

However, at least in the case of our model, the predictive skill is nearly completely lost when we use more of the available observational information about flux emergence in the photosphere, particularly concerning the emergence latitudes of bipolar magnetic regions. If we allow for the overlapping of cycles and use the full information provided by the butterfly diagram, the predictor largely reflects the strength of the ongoing cycle and exhibits almost no relation to the amplitude of the next cycle.

We have shown that this puzzling result, i.e., the schematic source providing predictive skill while the source based on actual data showing none, can be understood by the strong dependence of the schematic source on the epochs of the sunspot minima. The overlapping of cycles whose rise time is anticorrelated their amplitude (the Waldmeier effect) naturally leads to a time shift of the minima that is strongly related to the strength of the following cycle, thus affecting the strength of the schematic source for low-latitude emergence in the declining phase of a cycle. We have demonstrated the importance of this effect by showing that such propagation of amplitude information from the next cycle into the minimum epochs allows us to 'predict' synthetic cycles with random amplitudes with about the same skill as for the actual sequence of sunspot cycles.

The amplitude-dependent minimum shift explains the results obtained with our flux transport model. Moreover, it could account for the (partial) success of a number of precursor methods (for a summary, see Hathaway et al. 1999). This includes the correlation of 
the sunspot maximum with the activity level during the preceding minimum and the anticorrelation with the length of the preceding cycle (Wilson et al. 1998; Solanki et al. 2002), which directly result from the amplitude-related shift of the minimum epoch (see Figure 10). Likewise, the anticorrelation of the skewness of the cycle profile with the amplitude of the subsequent cycle (Lantos 2006) can be understood by the minimum shift: a weak follower cycle is preceded by a late minimum, so that the foregoing cycle has a longer decay time and thus becomes more asymmetric, and vice versa.

The amplitude-dependent minimum shift also explains the predictive skill of some geomagnetic precursors, such as the minimum level of the aa index (Ohl 1966). Other precursor methods are not so easily reduced to the minimum effect. For instance, when considering cycles 12-22 in retrospect, the method of Thompson (1993) yields a coefficient of 0.97 between the number geomagnetically disturbed days $(A p>25)$ during cycle $n$ with the sum of the sunspot number maxima of cycle $n$ and $n+1$ (Hathaway et al. 1999). For cycle 23, however, this method predicted a maximum (yearly) sunspot number of about 160, more than $30 \%$ too large. It is therefore unclear whether this method has a real physical basis or if the good correlation in the past is just fortuitous, the method being just one of a large number of other possibilities and happened to provide a large correlation coefficient for the existing data. Some methods depending on a ad-hoc recipes for the selection or division of geomagnetic data during the declining phase of the sunspot cycle into an 'activity-related' and an 'interplanetary' component (Legrand \& Simon 1981; Fevnman 1982) have also been shown to provide high correlations for past cycles (e.g., Hathaway \& Wilson 2006). They may capture early manifestations of the (extended) new cycle in middle to high latitudes, for instance, in the form of ephemeral active regions (Harvey 1992), but the physical connection remains to be understood. In any case, it appears to be the early influence of the new cycle which makes a prediction possible.

In summary, we have shown that the predictive skill of many precursor methods and that of our simple flux transport model with a schematic source can be reduced to the time shift of solar minima depending on the strength of the next cycle. Therefore, information about the next cycle is available (in statistical sense, of course) at the time when the solar minimum is clearly identified - not always an easy and straightforward task, see Harvey \& White (1999). Concerning our physical understanding of the solar cycle and the dynamo mechanism, the crucial point is that the predictive skill of such precursors does neither require nor imply a physical relation between surface manifestations of subsequent cycles - we have shown that such methods can be applied to for random sequences of cycles with fully independent amplitudes.

In any case, one should not be misled by high correlation coefficients for reproducing the 
past because most methods can be adjusted once the actual numbers are known. At best, precursor methods could indicate a trend for the next cycle, but we cannot expect to fully capture the intrinsic variability of the solar dynamo process with such simple recipes. Our work gives no indication that flux-transport models of the surface field could improve the predictions, quite the contrary: our model shows no predictive skill when the actually observed emergence latitudes are used. There is no reason to assume that a more sophisticated 2D surface flux transport model would lead to a different result. The predictive skill arising in the case of a schematic prescription of the emergence latitudes results from the shift of the minimum epoch depending on the strength of the next cycle, i.e., on information from the cycle to be predicted. We can tune the parameters of the model to obtain correlation coefficients with past cycles of $r \simeq 0.95$, but much simpler precursors (like the activity level 3 years before minimum) also reach values around 0.90 .

While lacking the sub-surface transport and the generation of the toroidal field, our flux-transport approach is conceptually similar to the model of Dikpati et al. (2006) and (Dikpati \& Gilman 2006). We conjecture that the amplitude-dependent minimum shift should have an impact on the predictive skill of their model as well. This could be easily tested by replacing the schematic latitude dependence of the source by the actual emergence latitudes for each sunspot group, thus avoiding the minimum shift effect.

Extended discussions with Mausumi Dikpati and Peter Gilman about the DGG model are gratefully acknowledged. Laura Balmaceda kindly put to our disposal her cross-calibrated dataset of sunspot group areas. Helpful comments by an anonymous referee led to a substantial improvement of the presentation in various parts of the paper.

\section{REFERENCES}

Arge, C. N., Hildner, E., Pizzo, V. J., \& Harvey, J. W. 2002, J. Geophys. Res. (Space Physics), 107, DOI 10.1029/2001JA000503

Babcock, H. W. 1961, ApJ, 133, 572

Balmaceda, L., Solanki, S. K., \& Krivova, N. 2005, Memorie della Societa Astronomica Italiana, 76, 929

Baumann, I., Schmitt, D., Schüssler, M., \& Solanki, S. K. 2004, A\&A, 426, 1075

Baumann, I., Schmitt, D., \& Schüssler, M. 2006, A\&A, 446, 307 
Choudhuri, A. R., Schüssler, M., \& Dikpati, M. 1995, A\&A, 303, L29

Dikpati, M. \& Charbonneau, P. 1999, ApJ, 518, 508

Dikpati, M., de Toma, G., \& Gilman, P. A. 2006, Geophys. Res. Lett., 33, 5102

Dikpati, M. \& Gilman, P. A. 2006, ApJ, in press

Durney, B. R. 1995, Sol. Phys., 160, 213

—. 1996, Sol. Phys., 166, 231

Durrant, C. J., Turner, J., \& Wilson, P. R. 2002, Sol. Phys., 211, 103

Durrant, C. J., Turner, J. P. R., \& Wilson, P. R. 2004, Sol. Phys., 222, 345

Durrant, C. J. \& Wilson, P. R. 2003, Sol. Phys., 214, 23

Echer, E., Rigozo, N., Nordemann, D., \& Vieira, L. 2004, Annales Geophysicae, 22, 2239

Feynman, J. 1982, J. Geophys. Res., 87, 6153

Giovanelli, R. G. 1985, Australian Journal of Physics, 38, 1045

Harvey, K. L. 1992, in The Solar Cycle, ed. K. L. Harvey (San Francisco: Astronomical Society of the Pacific, ASP Conf. Series Vol. 27), 335

Harvey, K. L. \& White, O. R. 1999, ApJ, 515, 812

Hathaway, D. H. \& Wilson, R. M. 2006, Geophys. Res. Lett., in press

Hathaway, D. H., Wilson, R. M., \& Reichmann, E. J. 1994, Sol. Phys., 151, 177

—. 1999, J. Geophys. Res., 104, 22375

—. 2002, Sol. Phys., 211, 357

Howard, R. F. 1991, Sol. Phys., 136, 251

Küker, M., Rüdiger, G., \& Schultz, M. 2001, A\&A, 374, 301

Lantos, P. 2006, Sol. Phys., 238, 199

Lantos, P. \& Richard, O. 1998, Sol. Phys., 182, 231

Layden, A. C., Fox, P. A., Howard, J. M., Sarajedini, A., \& Schatten, K. H. 1991, Sol. Phys., 132,1 
Legrand, J. P. \& Simon, P. A. 1981, Sol. Phys., 70, 173

Leighton, R. B. 1969, ApJ, 156, 1

Li, K. 1999, A\&A, 345, 1006

Ohl, A. I. 1966, Soln. Dann., 12, 84

Rigozo, N. R., Echer, E., Vieira, L. E. A., \& Nordemann, D. J. R. 2001, Sol. Phys., 203, 179

Schüssler, M. \& Baumann, I. 2006, A\&A, in press

Schatten, K. H. 2003, Adv. Space Res., 32, 451

Schatten, K. H., Scherrer, P. H., Svalgaard, L., \& Wilcox, J. M. 1978, Geophys. Res. Lett., 5,411

Schrijver, C. J. 2001, ApJ, 547, 475

Schrijver, C. J., DeRosa, M. L., \& Title, A. M. 2002, ApJ, 577, 1006

Sello, S. 2001, A\&A, 377, 312

Solanki, S. K., Krivova, N. A., Schüssler, M., \& Fligge, M. 2002, A\&A, 396, 1029

Svalgaard, L., Cliver, E. W., \& Kamide, Y. 2005, Geophys. Res. Lett., 32, 1104

Thompson, R. J. 1993, Sol. Phys., 148, 383

van Ballegooijen, A. A., Cartledge, N. P., \& Priest, E. R. 1998, ApJ, 501, 866

Waldmeier, M. 1935, Astron. Mitt. Eidgen. Sternw. Zürich, 14, 105

Wang, Y.-M., Lean, J., \& Sheeley, N. R. 2002, ApJ, 577, L53

Wang, Y.-M., Lean, J. L., \& Sheeley, N. R. 2005, ApJ, 625, 522

Wang, Y.-M., Nash, A. G., \& Sheeley, N. R. 1989a, ApJ, 347, 529

-. 1989b, Science, 245, 712

Wang, Y.-M. \& Sheeley, N. R. 1991, ApJ, 375, 761

Wilson, R. M., Hathaway, D. H., \& Reichmann, E. J. 1998, J. Geophys. Res., 103, 6595 

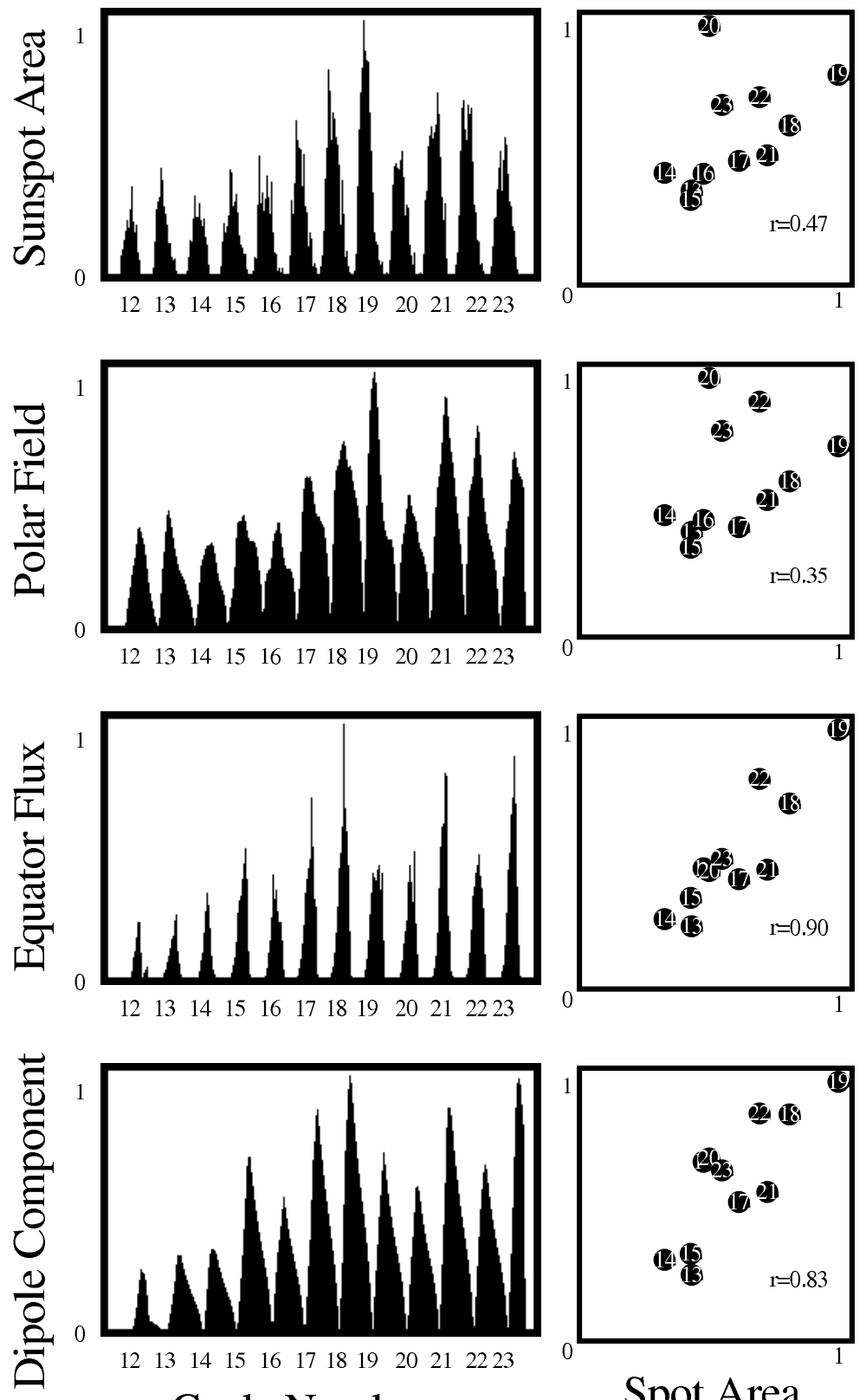

Cycle Number

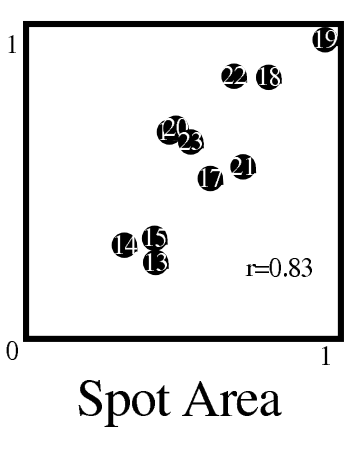

Fig. 1. - Time evolution of cycle-related quantities (left panels) and corresponding correlation diagrams (right panels). Top row: RGO/SOON sunspot areas and correlation diagram between subsequent maxima. Second row: unsigned polar field resulting from the surface flux transport model and correlation diagram of the maximum with the maximum spot area of the subsequent cycle. Third row: same for the magnetic flux diffusing over the equator per unit time from the flux transport model. Bottom row: same for the dipole component of the surface field from the flux transport model. The flux transport model is adapted as far as possible to the procedures and parameters used in the model of Dikpati et al. (2006). All quantities shown are normalized to their respective global maxima. The numbers of the 'predicted' cycles and the correlation coefficients are indicated in the correlation diagrams. Unless stated otherwise, all time-dependent quantities in this and the following diagrams are binned in six-month totals after applying a time-centered 6-month boxcar averaging. 


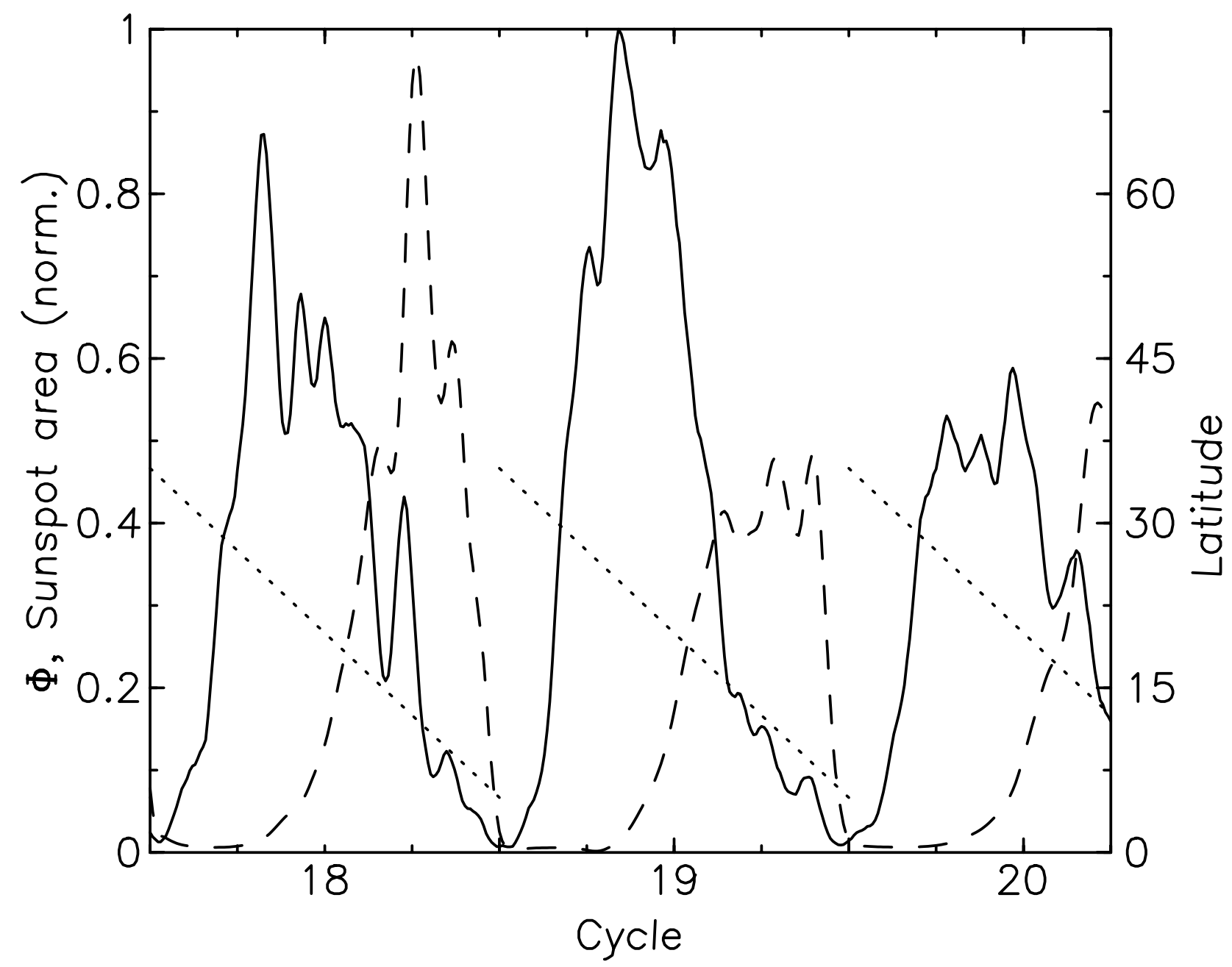

Fig. 2.- Normalized observed sunspot area (solid) and calculated magnetic flux diffusing over the equator ( $\Phi$, dashed) for cycles 18 to 20, together with the latitude progression of the centroid of the source term representing flux emergence in the model (dashed, scale to the right). The $\Phi$ curve peaks during the decling phases of the cycles, a few years before the minimum epochs. Their amplitude is determined by the level of low-latitude sunspot activity (flux emergence) during this phase. 


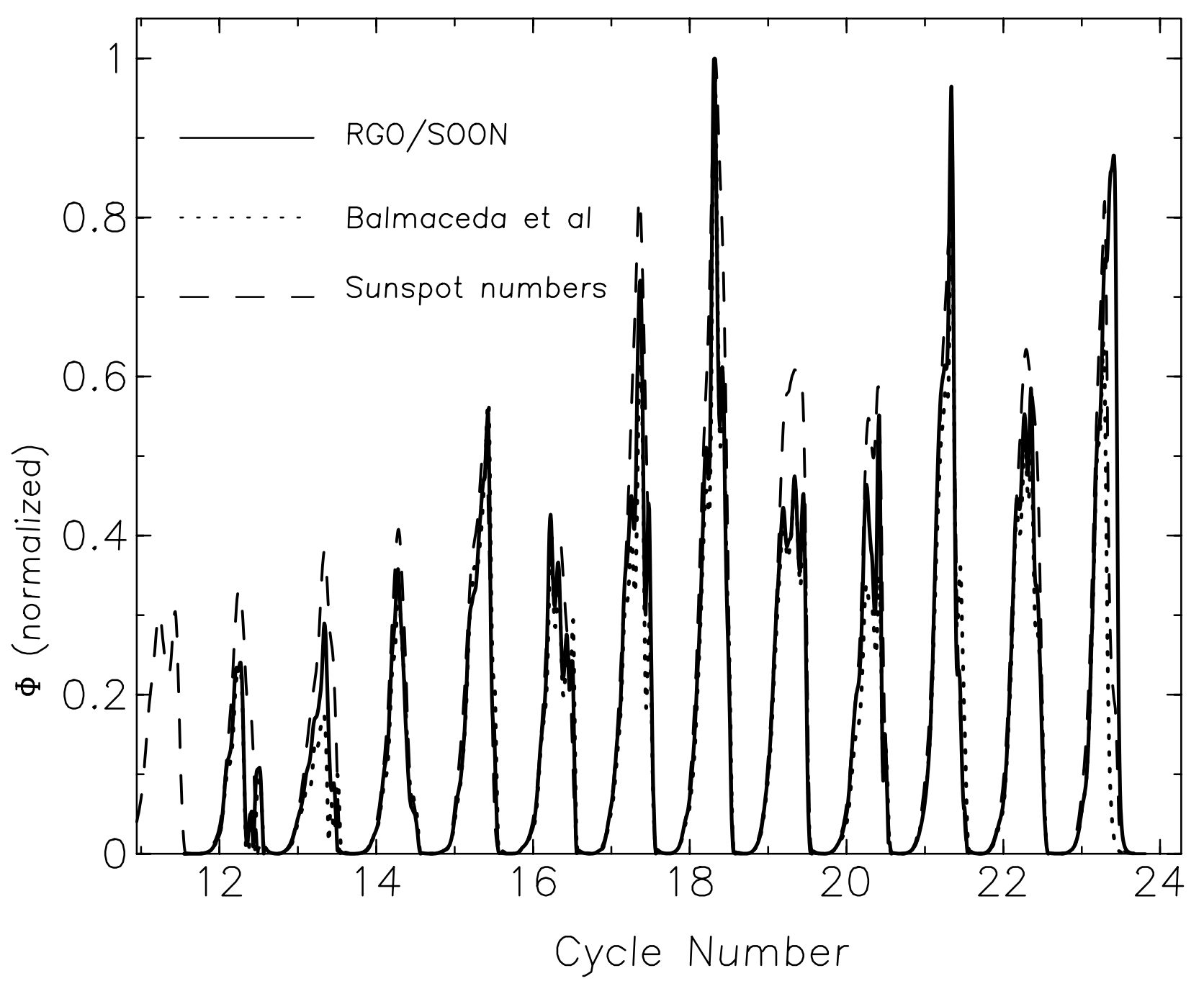

Fig. 3.- Magnetic flux diffusing over the equator per unit time $(\Phi)$, determined from the surface flux transport model with input from three different data sets. Solid line: RGO/SOON data according to D. Hathaway. Dotted line: cross-calibrated RGO, Russian and SOON data according to Balmaceda et al. (2005). Dashed line: monthly sunspot numbers. All curves are normalized to their respective maximum values. The parameters and procedures in the flux transport simulation are identical to those used for Figure 1. The correlation coefficients between the maxima of $\Phi$ and the activity maxima of the subsequent cycles are 0.90 (RGO/SOON), 0.87 (Balmaceda et al. 2005), and 0.73 (sunspot numbers), respectively. 


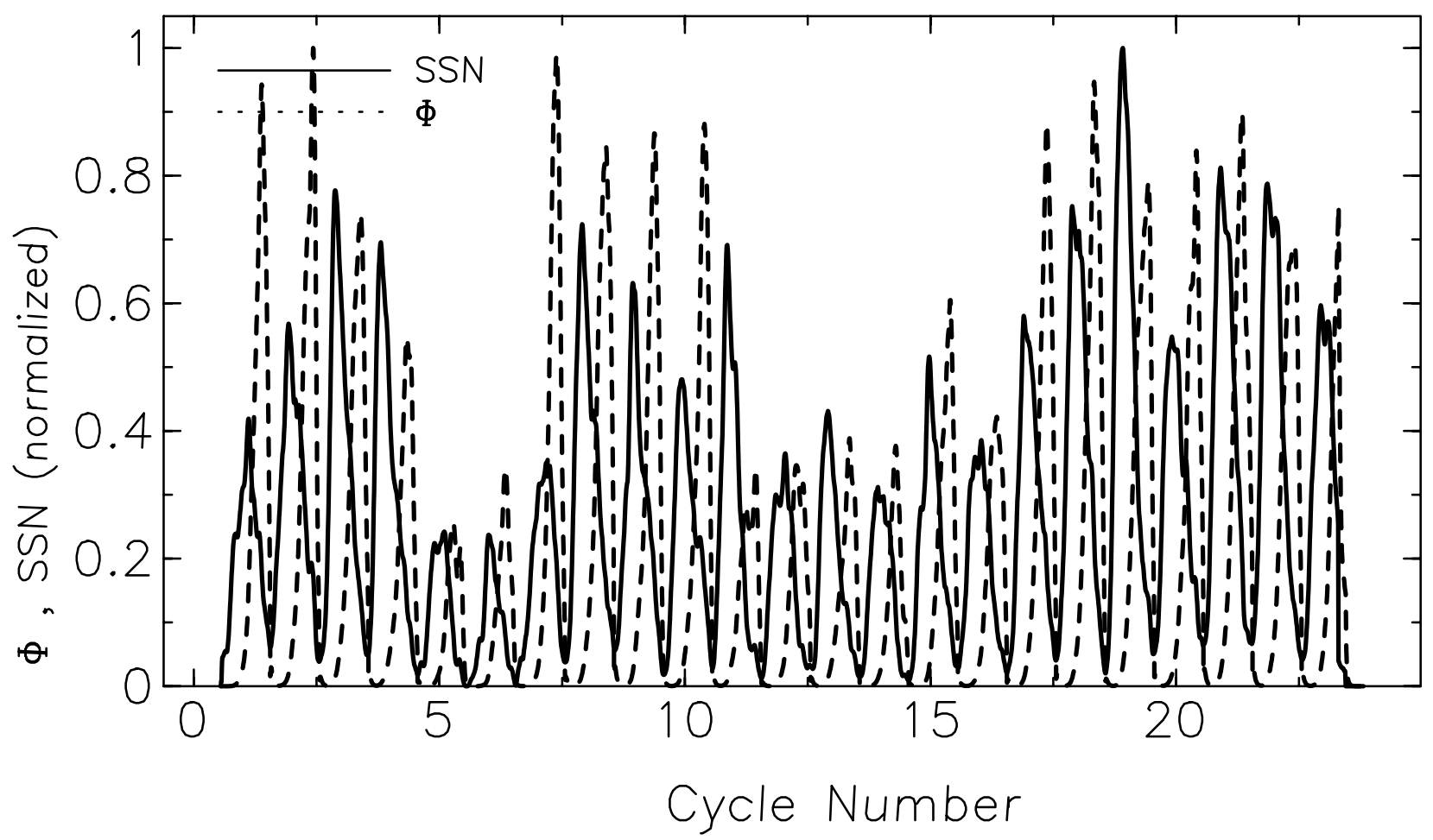

Fig. 4.- Magnetic flux diffusing over the equator ( $\Phi$, dashed curve) from the surface flux transport model with input source based upon monthly sunspot numbers for cycles $1-23$ (SSN, solid line). Both curves are normalized to their respective maximum values. The correlation coefficient between the maxima of $\Phi$ and the maximum sunspot numbers of the subsequent cycles is $r=0.80$. The cross-correlation of both curves reaches a maximum for a forward shift of the $\Phi$ curve by 6.75 years. 


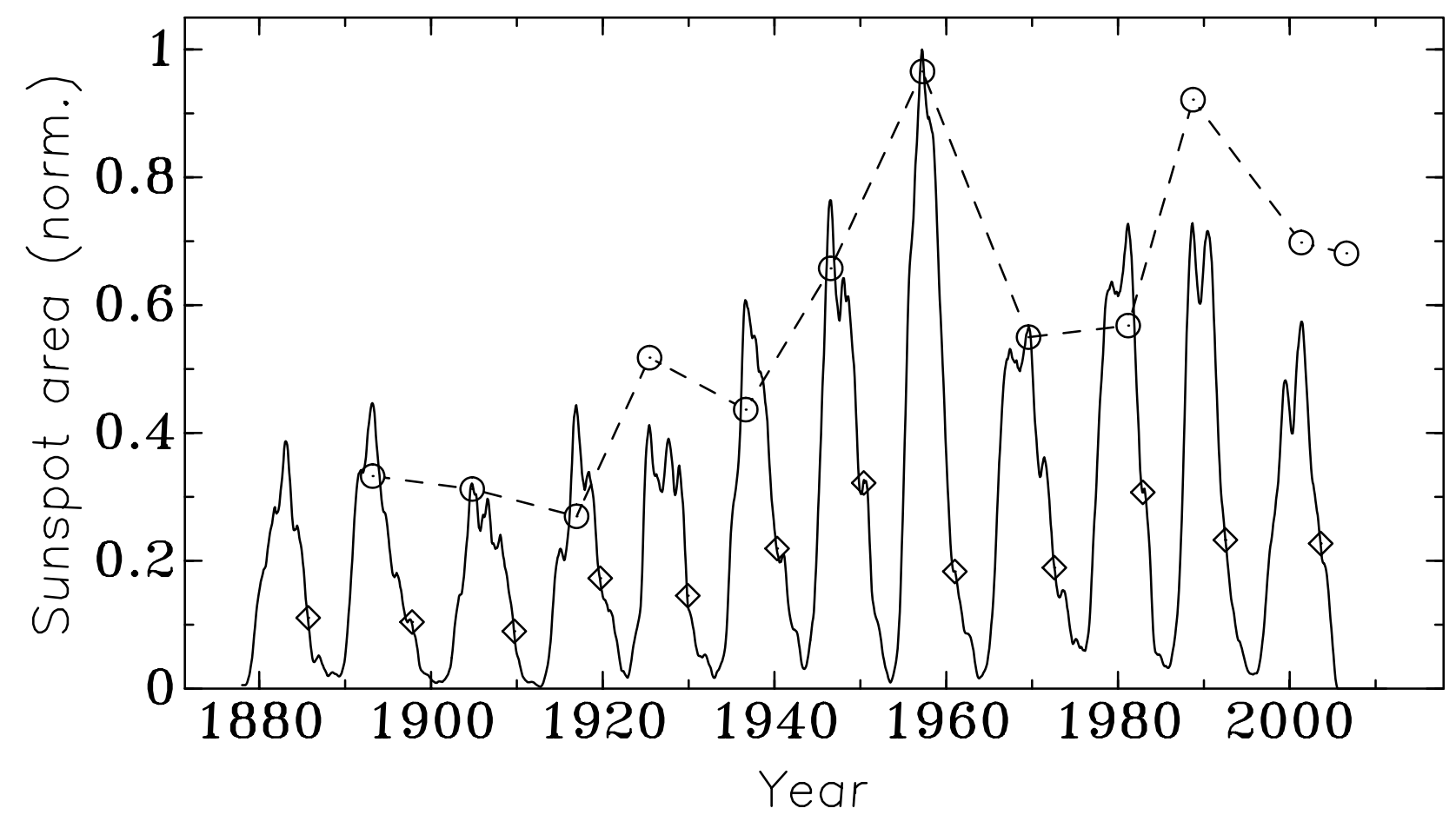

Fig. 5.- The activity level three years before sunspot minimum as a precursor for the amplitude of the subsequent cycle. The solid curve shows the normalized observed sunspot areas for cycles 12-23. The precursor is indicated by diamonds. In order to illustrate the predictive skill, open circles (connected by the dashed lines) representing 3 times the predictor value have been placed near the maximum epoch of the following cycles. The correlation coefficient between the predictor and the maximum sunspot area of the subsequent cycle is $r=0.84$. 


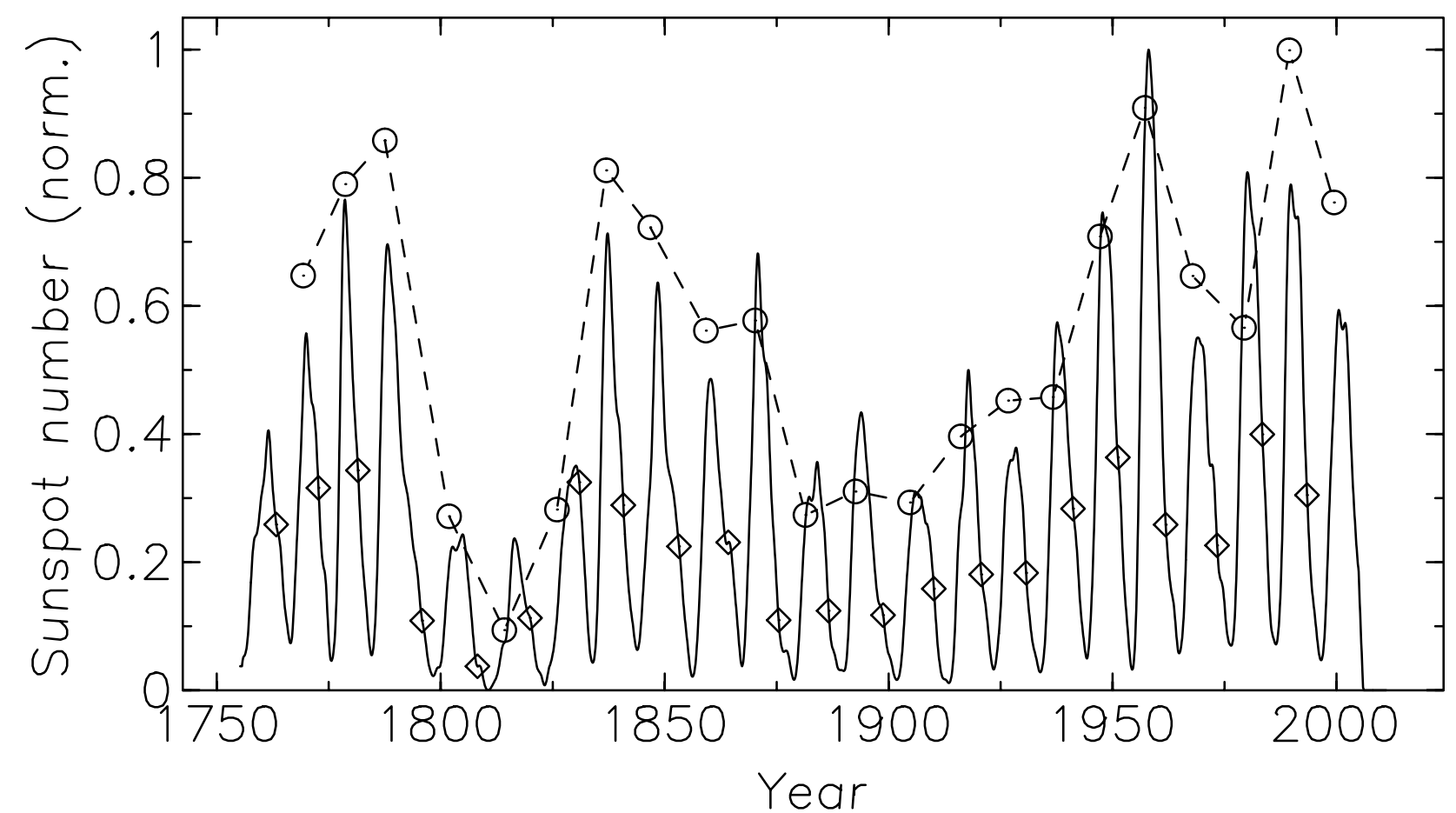

Fig. 6. - Same as Figure 5 with the sunspot numbers for cycles 1-23. The correlation coefficient is $r=0.89$. 


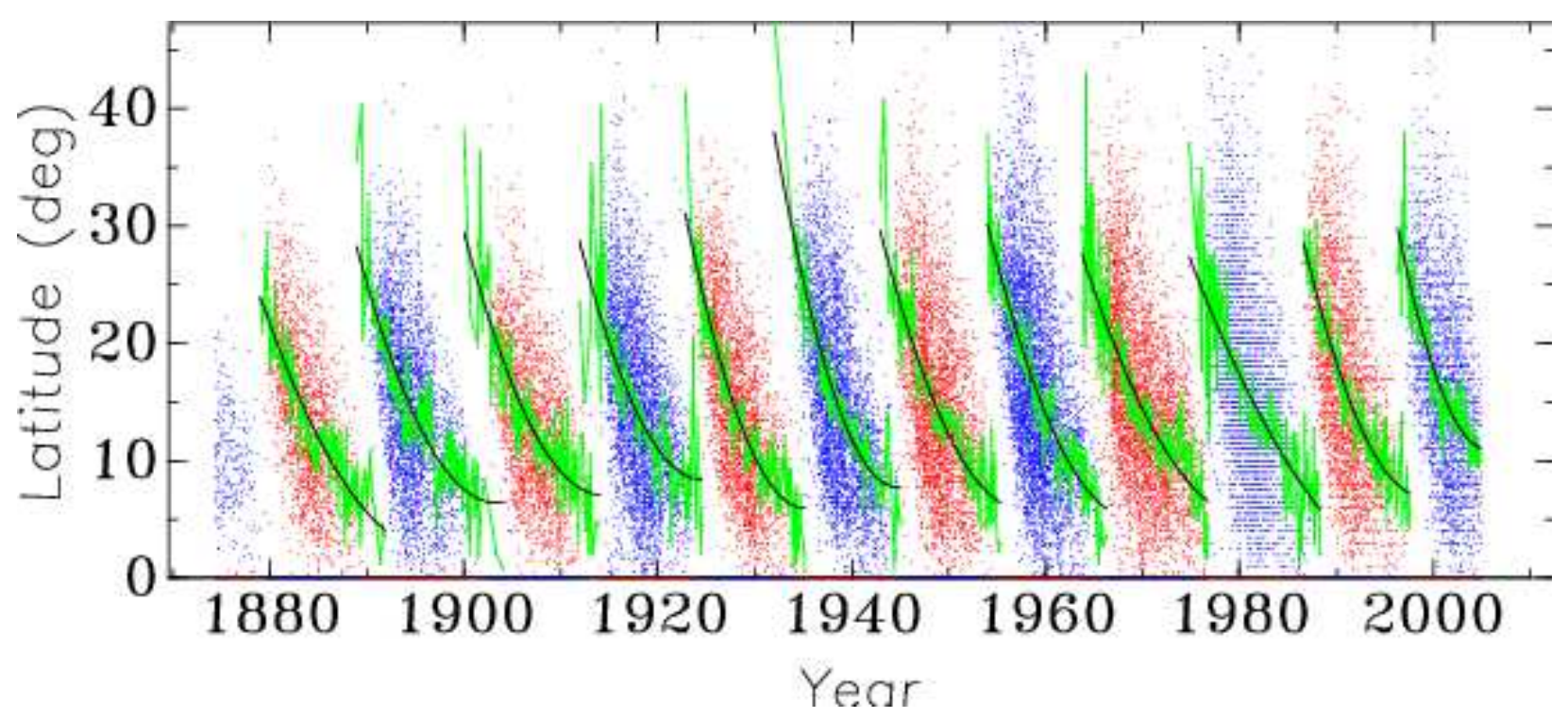

Fig. 7.- Emergence latitudes of sunspot groups for cycles 12-23. The blue and red dots indicate the individual sunspot groups as determined from the cross-calibrated data set of Balmaceda et al. (2005). The green curves show monthly averages, weighted with the corresponding group areas, and the black lines are parabolic fits of the green curves. The fit curves are only drawn for the time intervals during which sunspots of the corresponding cycle have been actually observed. 


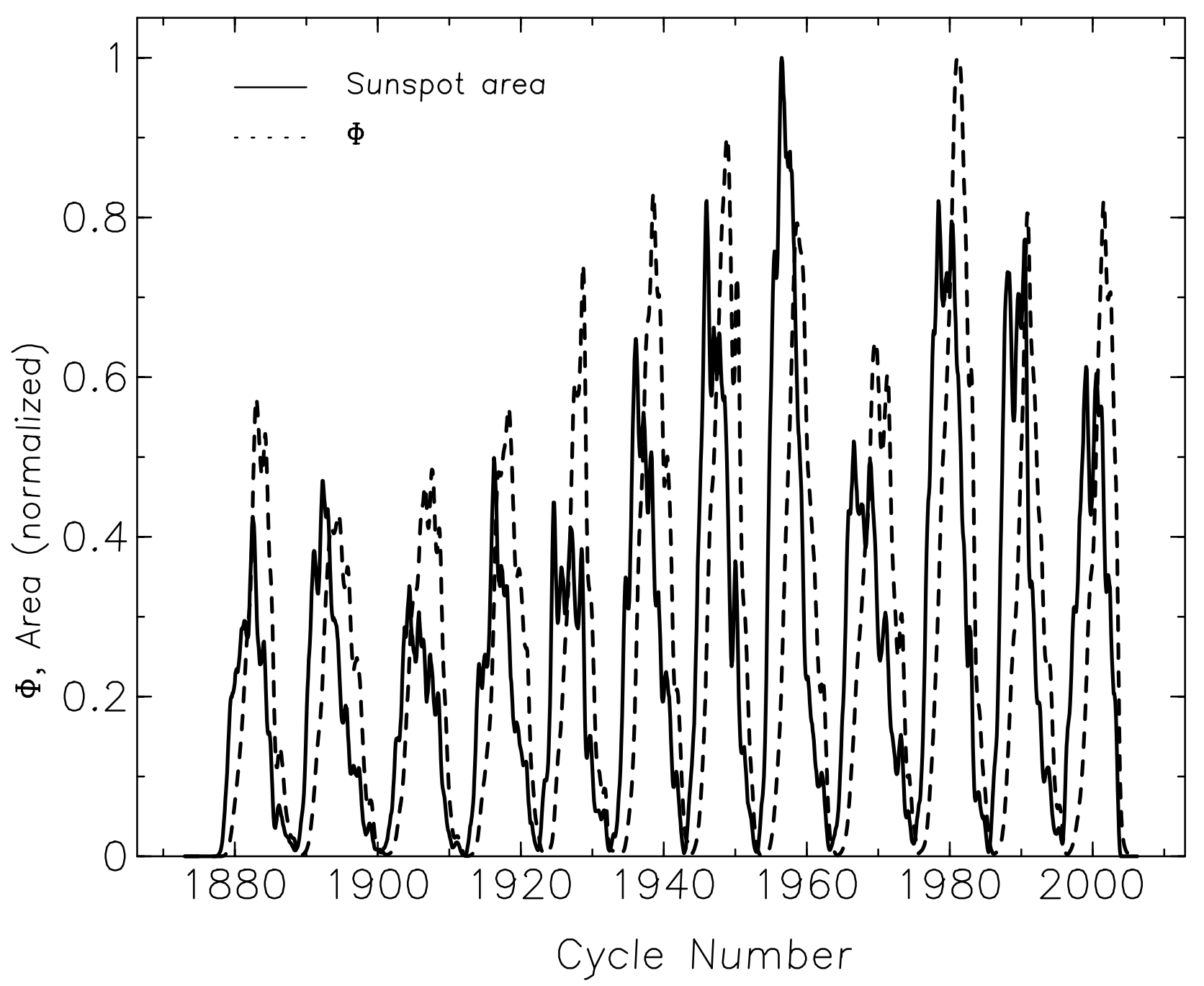

Fig. 8.- Comparison between the predictor quantity $\Phi$, the flux diffusing over the equator (dashed curve), and the observed sunspot area for cycles 12-23. $\Phi$ has been calculated with the flux-transport model with source input governed by the parabolic profiles of emergence latitudes shown in Figure 7. The correlation coefficient between the maxima of the $\Phi$ and the maxima of the sunspot area of the subsequent cycle is $r=0.75$. 


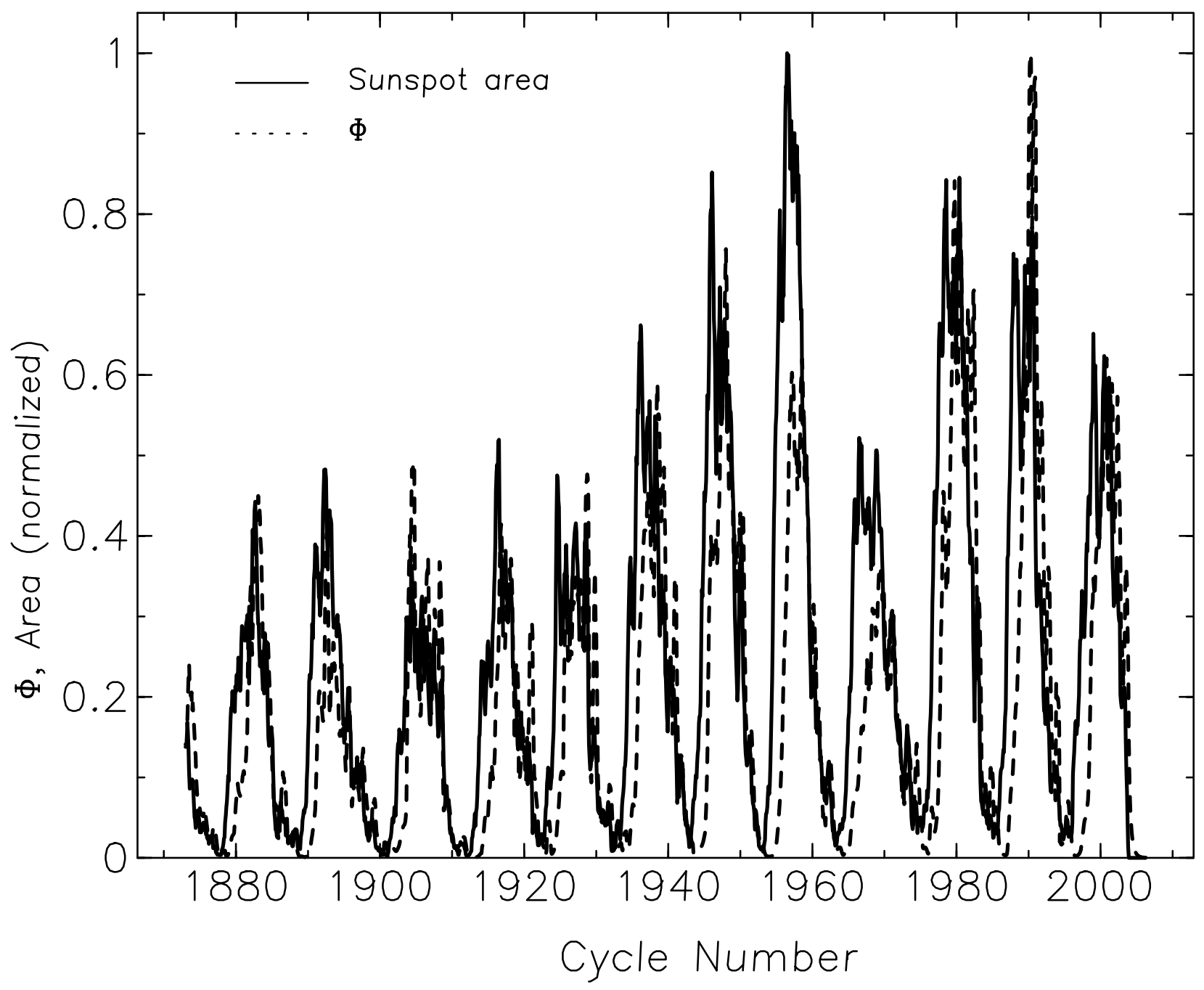

Fig. 9.- Same as Figure 8 for a source term based upon the emergence of each individual sunspot group in the dataset of Balmaceda et al. (2005). The correlation coefficient between the maxima of the $\Phi$ and the maxima of the sunspot area of the subsequent cycle is $r=0.33$. 

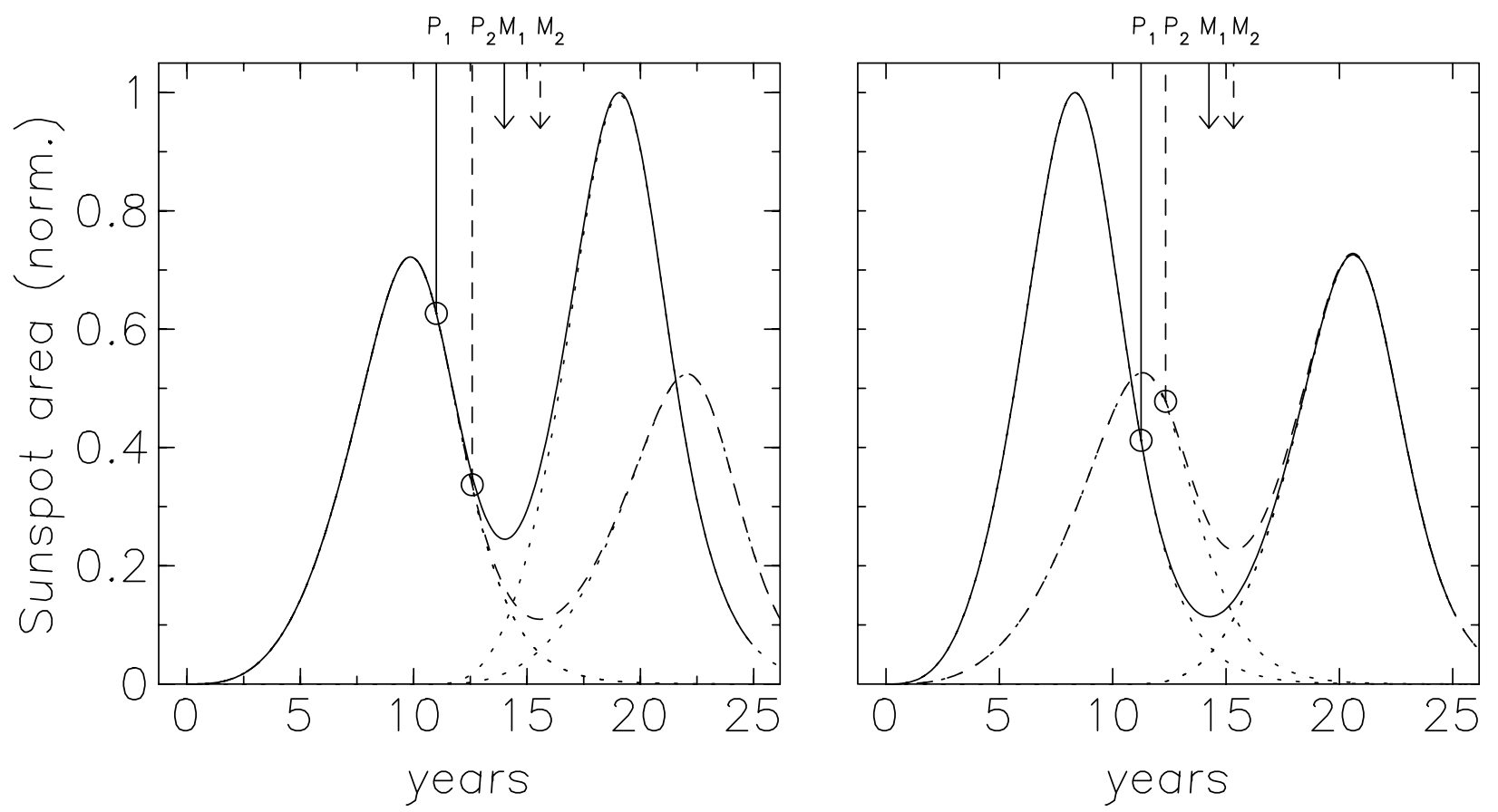

Fig. 10. - Schematic illustration of the amplitude-dependent shift of the minima between overlapping sunspot cycles and its influence on precursor quantities. Left panel: effect of the following cycle. A stronger follower cycle (solid curve) with a shorter rise time leads to an earlier minimum (M1) and a higher predictor (P1) than a weaker subsequent cycle with a longer rise time (dashed curve, minimum M2, predictor P2). Right panel: effect of the preceding cycle. Since the cycle asymmetry mainly affects the rising phase, the influence of the strength of the preceding cycle on the minimum epoch and on the predictor is much smaller. 


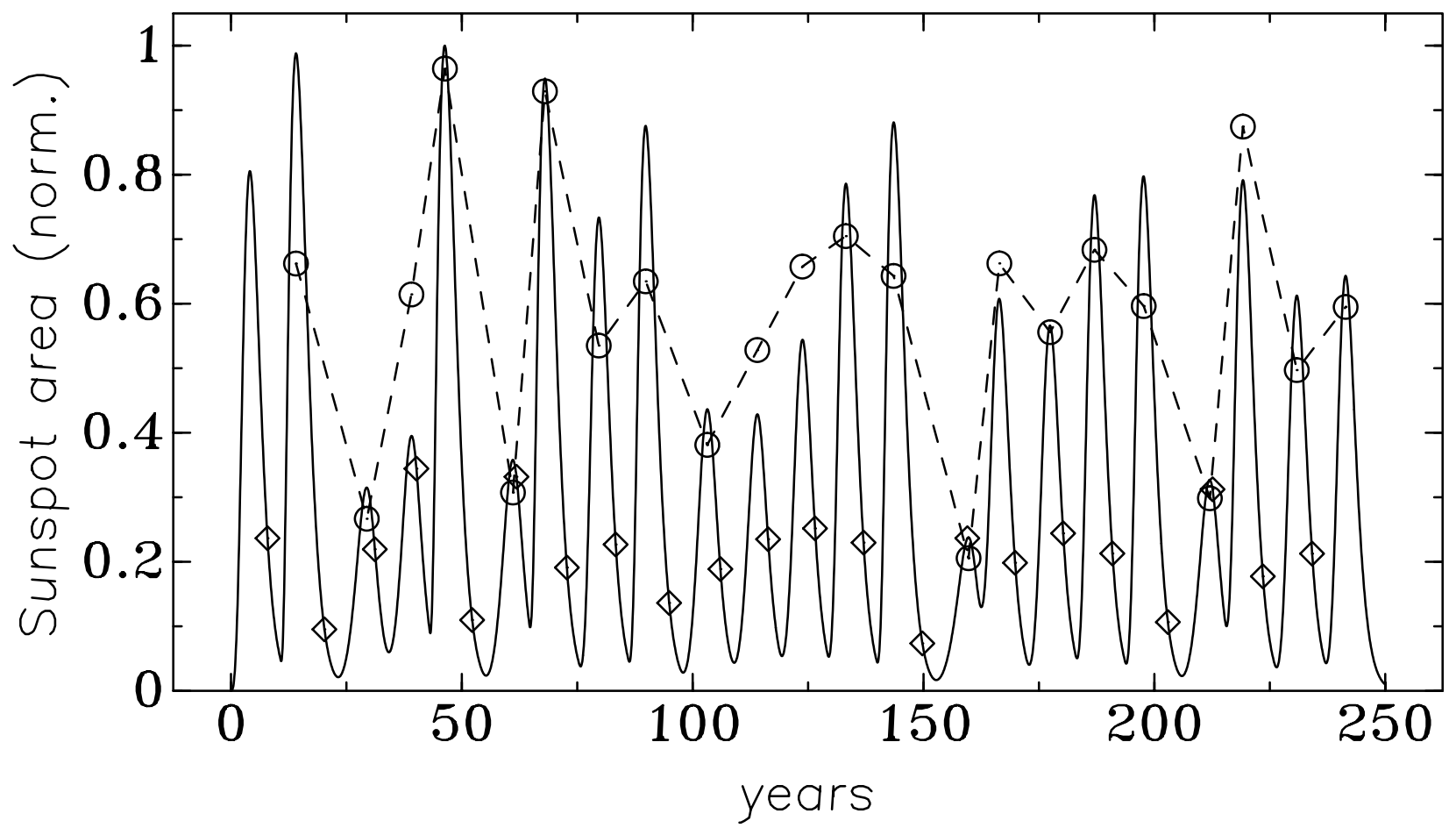

Fig. 11.- Comparison of the activity three years before minimum with the maximum of the subsequent cycle for a series of 23 synthetic solar cycles with a random distribution of maximum sunspot area between 500 and 3000 millionths of a solar hemisphere. The quality of the 'prediction' of the random amplitudes (correlation coefficient $r=0.84$ ) is comparable to that based on the real data (see Figure [6 with $r=0.89$ ). 


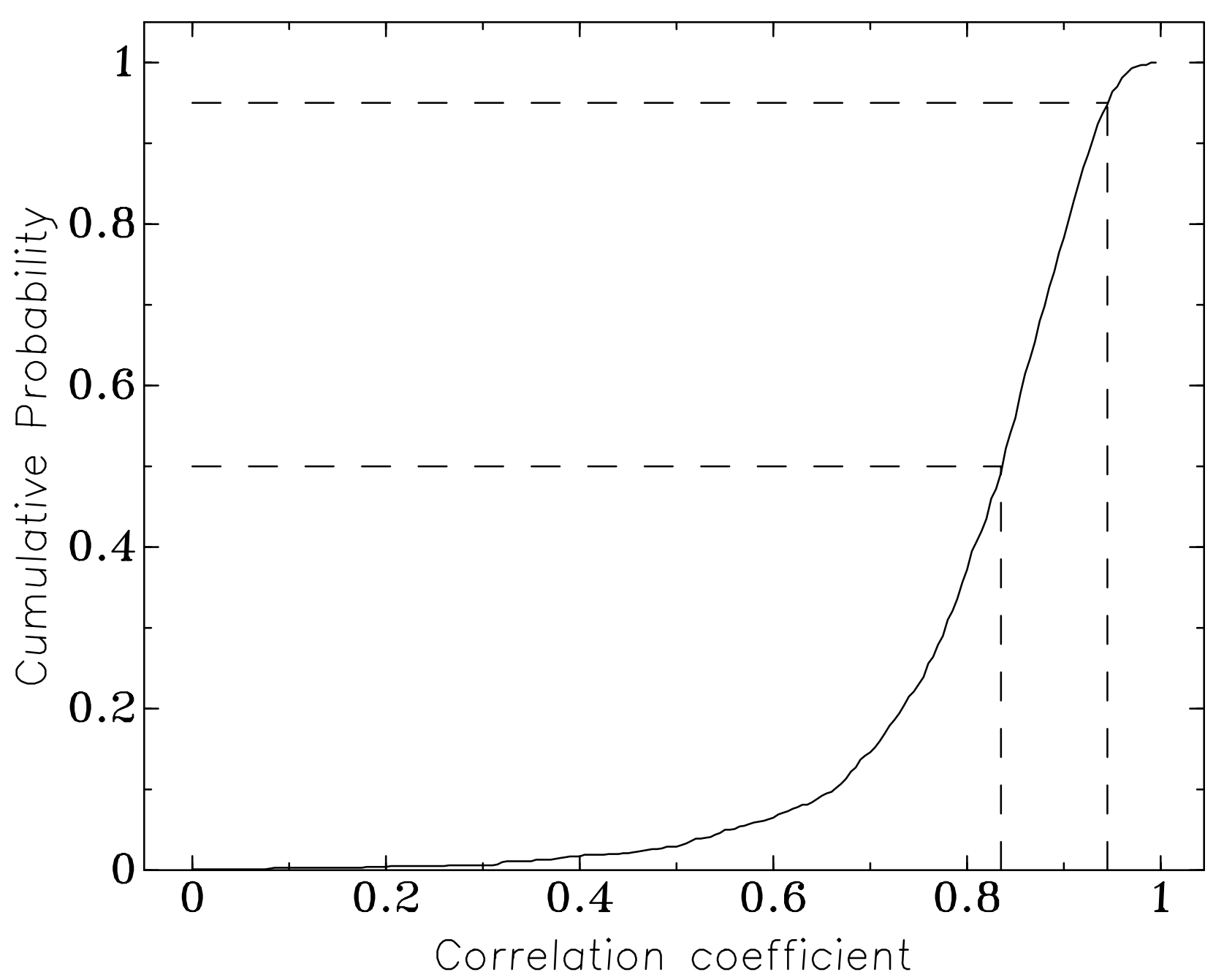

Fig. 12.- Cumulative probability distribution function for the correlation coefficients between the maximum sunspot number of a cycle and the sunspot number three years before the preceding minimum for 1000 synthetic datasets of 8 cycles each. The cycles have random amplitudes between 500 and 3000 millionths of a solar hemisphere. The correlation coefficient is larger than 0.83 in $50 \%$ of the cases and exceeds 0.95 in $5 \%$ of the cases. 